\title{
SELECTING Experts USINg DATA QUALITY CONCEPTS
}

\author{
Carlos Eduardo Barbosa ${ }^{1}$, Marcio Antelio ${ }_{3}^{1,2}$, Jano Moreira de Souza ${ }^{1}$, Jonice \\ Oliveira $^{3}$ \\ ${ }^{1}$ COPPE, Universidade Federal do Rio de Janeiro (UFRJ), Rio de Janeiro, Brazil \\ ${ }^{2}$ CEFET, Rio de Janeiro, Brazil \\ ${ }^{3}$ DCC, Universidade Federal do Rio de Janeiro (UFRJ), Rio de Janeiro, Brazil
}

\begin{abstract}
Personal networks are not always diverse or large enough to reach those with the right information. This problem increases when assembling a group of experts from around the world, something which is a challenge in Future-oriented Technology Analysis (FTA). In this work, we address the formation of a panel of experts, specifically how to select a group of experts from a huge group of people. We propose an approach which uses data quality dimensions to improve expert selection quality and provide quality metrics to the forecaster. We performed a case study and successfully showed that it is possible to use data quality methods to support the expert search process.
\end{abstract}

\section{KEYWORDS}

Learning Curves, Knowledge Depreciation,Data Quality,Expert Selection, Decision-making, Futureoriented Technology Analysis.

\section{INTRODUCTION}

Nowadays, budget constraints make it necessary to choose which scientific research works receive priority. Therefore, it has become necessary to develop ways to identify long-term technologies that will have the greatest impact on the future of science, the economy, and society. Several approaches for predicting the future of technology have been united under the term future-oriented technology analysis (FTA).

There are several FTA methods which dependon experts' opinions. Porter et al. [1] listed Delphi, Focus Groups, Interviews, and Participatory Techniques. These methods use a large group of experts, relying on the 'wisdom of crowds' [2].

In the other hand, Data quality techniques may be used to measure the expert search quality of the results. Uotila and Melkas [3] discussed the similarities and the differences between data quality, information, and knowledge. In the scope of this work, the terms 'data' and 'information' are interchangeable.

The purpose of this work is to provide a framework based on learning curves and knowledge depreciation to form a panel of experts, and to measure its intrinsic quality using data quality dimensions. To illustrate the framework, a small Delphi modification was performed.

In Section 2, a literature review is done, which covers FTA, learning curves, knowledge depreciation, and data quality concepts; Section 3 presents the expert selection framework; Section 4 presents a case study which shows the framework in practice; and Section 5 discusses the results and conclusions.

DOI : 10.5121/ijdms.2015.7601 
International Journal of Database Management Systems ( IJDMS ) Vol.7, No.6, December 2015

\section{LITERATURE REVIEW}

\subsection{Future-oriented Technology Analysis}

Future-oriented technology analysis (FTA) is an umbrella concept which encompasses any systematic methodology for supporting decisions related to emerging technology, including its development and future impacts [1]. For a short time, FTA was known as technology futures analysis (TFA), but its name was later altered to give the idea of future-oriented, not technologyoriented [4]. The FTA concept is an evolution of two terms which describe the FTA facets: technology foresight and technology forecasting.

The military is heavily involved with FTA, since war efforts have resulted in advances in several areas of knowledge; for example, the nuclear, computing, internet, and space domains [5]. In the 1950s and 1960s, FTA was used by the military in the Cold War as a tool for planning and prioritizing the research and development of military technology, something which involves long development times. Today, commercial technological competitors use FTA as a tool for business intelligence. Competitive technological intelligence is concerned with forecasting the technology developments of others in order to constrain corporate research and development. National technology foresight, as well as industry or product technology roadmaps, show that industry and government are interested in planning, prioritizing, and evaluating their research and development programs [6].

\subsection{Learning curves}

Wright [7] noted that labour costs for production are progressively reduced in a non-linear way as the production becomes cumulative. This may be expressed by a curve, defined by Argote and Epple [8]:

$$
y=a x^{-b}
$$

In which:

- $y$ is the labour time for the $\mathrm{x}^{\text {th }}$ unit;

- $a$ is the labour time for the first unit;

- $x$ is the number of units; and

- $\quad b$ is the learning rate (i.e., rate for reduction in labour time).

According to Dorroh et al. [9], cost researchers have noted that simple log-linear curve models are inadequate, and so they proposed several alternative learning curve specifications to address this inadequacy. For this reason, in this work, the learning curve model is inspired by a Gompertz function [10].

Empirical studies have shown that unit costs do not continue to decline indefinitely with cumulative knowledge, a phenomenon that is referred to as the plateau effect [11].

\subsection{Knowledge Depreciation}

Knowledge depreciation (KD) refers to the erosion in the knowledge inside organizations or individuals. In organizations, employee turnover may lead to loss of knowledge and learning [11]. When leaving an organization, the worker carries his unique knowledge, thus depreciating the organization's knowledge. The KD rate varies widely across industrial settings. Knowledge management initiatives may be used to resist this depreciation [12]. At the individual level, there 
International Journal of Database Management Systems ( IJDMS ) Vol.7, No.6, December 2015

is also evidence of KD [11]. Learning new skills may provoke unlearning in others. Temporal memory decay is the major factor that results in forgetfulness [13].

\subsection{Quality of data, information, and knowledge}

According to Uotila and Melkas [3], data refers to the most basic descriptive element which represents a perception or measurement, lacking in content, meaning, or intent. Information is more than a set of data; it is the output of a process that interprets and manipulates data. Knowledge consists of an assortment of inputs: information, experiences, beliefs, relationships, and techniques, that an individual mentally synthesizes together to determine what a specific situation means and how to handle it. The terms and their meanings are shown in Table 1.

Table 1. Definition of terms, from [3].

\begin{tabular}{|l|l|}
\hline Term & Meaning \\
\hline Data & $\begin{array}{l}\text { A collection of attribute values about some entity or event organized as a } \\
\text { record and stored in some type of file structure (e.g., relational table). }\end{array}$ \\
\hline Information & $\begin{array}{l}\text { The output that results when a specified process manipulates raw source } \\
\text { data and semi-processed component data items into a predefined format. }\end{array}$ \\
\hline $\begin{array}{l}\text { Knowledge (of } \\
\text { an area) }\end{array}$ & $\begin{array}{l}\text { A process by which, justified true beliefs about relationships between ideas, } \\
\text { information, and experiences relevant to a particular area are used to } \\
\text { generate situational awareness. }\end{array}$ \\
\hline $\begin{array}{l}\text { Data quality, } \\
\text { information } \\
\text { quality, and } \\
\text { knowledge } \\
\text { quality }\end{array}$ & $\begin{array}{l}\text { Data, information products, and knowledge are of high quality if they are fit } \\
\text { for their intended use in conducting business operations, decision making, } \\
\text { and planning. }\end{array}$ \\
\hline
\end{tabular}

A user's perception of what is accurate, current, important, or useful is also determined by why they seek it [14]. Similarly to search engine algorithms, a single query may convey different meanings or goals, and better results could be achieved with a better understanding of the intention of a query [15].

The widely accepted information quality frameworks vary in approach and application, but they all share several characteristics regarding their classifications of quality dimensions [15]. Data quality is multidimensional and may be described according to the quality of the values collected [3]. When discussing data quality dimensions, it is reasonable to replace data with information or knowledge without losing the meaning [3]. Common methods to identify the quality dimensions associated with data are shown in Table 2.

Table 2. Methods to identifying data quality dimensions, from [3].

\begin{tabular}{|c|l|c|}
\hline Approach & Definition & Example \\
\hline Intuitive & $\begin{array}{l}\text { The selection of quality attributes in a specific study is } \\
\text { based on the individual's experience or intuitive } \\
\text { understanding about what attributes are important. }\end{array}$ & {$[16]$} \\
\hline Systematic & $\begin{array}{l}\text { Focuses on how the output of a process may become } \\
\text { deficient. }\end{array}$ & {$[17]$} \\
\hline Empirical & $\begin{array}{l}\text { Collects input from consumers to determine the } \\
\text { characteristics they use to assess whether the end product } \\
\text { is fit for use in their tasks. }\end{array}$ & {$[18]$} \\
\hline
\end{tabular}

Wang and Strong [18] proposed a hierarchical conceptual framework, in which groups of data quality dimensions are labelled. These representative labels capture the essence of the dimensions 
from the data quality groups proposed: intrinsic, contextual, representational, and accessible. A summary of the common information quality dimensions and their short definitions are shown in Table 3.

Intrinsic data quality includes accuracy, objectivity, believability, and reputation. This suggests that accurate and objective data also needs believability and reputation in order to be considered to be high quality data. This is somewhat analogous to product quality [18].

Table 3.Common dimensions of data quality, from [15].

\begin{tabular}{|c|c|}
\hline Dimension & Definition \\
\hline Accuracy & Extent to which data are correct, reliable, and certified as free of error. \\
\hline Consistency & $\begin{array}{l}\text { Extent to which information is presented in the same format and is compatible } \\
\text { with previous data. }\end{array}$ \\
\hline Security & $\begin{array}{l}\text { Extent to which access to information is appropriately restricted to maintain its } \\
\text { security. }\end{array}$ \\
\hline Timeliness & Extent to which the information is sufficiently up-to-date for the task at hand. \\
\hline Completeness & $\begin{array}{l}\text { Extent to which information is not missing and is of sufficient breadth and } \\
\text { depth for the task at hand. }\end{array}$ \\
\hline Conciseness & $\begin{array}{l}\text { Extent to which information is concisely represented without being } \\
\text { overwhelming (i.e., brief in presentation, yet complete and to the point). }\end{array}$ \\
\hline Reliability & Extent to which information is correct and reliable. \\
\hline Accessibility & Extent to which information is available, or quick and easy to retrieve. \\
\hline Availability & Extent to which information is physically accessible. \\
\hline Objectivity & Extent to which information is unbiased, unprejudiced, and impartial. \\
\hline Relevancy & Extent to which information is applicable and helpful for the task at hand. \\
\hline Usability & Extent to which information is clear and easily able to be used. \\
\hline Understandability & $\begin{array}{l}\text { Extent to which data are clear and unambiguous and can be easily } \\
\text { comprehended. }\end{array}$ \\
\hline Amount of data & Extent to which the quantity or volume of available data is appropriate. \\
\hline Believability & Extent to which information is regarded as true and credible. \\
\hline Navigation & Extent to which data are easily found and linked to. \\
\hline Reputation & Extent to which information is highly regarded in terms of source or content. \\
\hline Usefulness & Extent to which information is applicable and helpful for the task at hand. \\
\hline Efficiency & $\begin{array}{l}\text { Extent to which data are able to quickly meet the information needs for the } \\
\text { task at hand. }\end{array}$ \\
\hline
\end{tabular}

Accessible data quality includes accessibility and security. Data consumers view this as an important aspect of data quality. Treating accessible data quality as a data quality category or incorporating its dimensions into other categories makes almost no difference. In any case, accessibility dimensions are indispensable [18].

Contextual data quality includes relevancy, value-addedness, timeliness, completeness, and amount of information. The contextual data quality shows that data quality must be analysed in the context of the current task [18].

Representational data quality includes concise representation, consistent representation, interpretability, and ease of understanding. These aspects are related to the data format and meaning. For data consumers, well-represented data must not only be concise and consistently represented, but also interpretable and easy to understand [18]. 
International Journal of Database Management Systems ( IJDMS ) Vol.7, No.6, December 2015

Naumann and Rolker [19] grouped the entities and factors involved in information quality into subject, object, and process criteria. The identified criteria and their assessment methods are shown in Table 4.

Table 4. Classification of information quality criteria, from [19].

\begin{tabular}{|l|l|l|}
\hline Assessment class & Criterion & Assessment method \\
\hline Subject criteria & Believability & User experience \\
\cline { 2 - 3 } & Concise representation & User sampling \\
\cline { 2 - 3 } & Interpretability & \\
\cline { 2 - 3 } & Relevancy & User sampling \\
\cline { 2 - 3 } & Reputation & Continuous user assessment \\
\cline { 2 - 3 } & Understandability & User experience \\
\hline & Value-addedness & User sampling \\
\hline Object criteria & Completeness & Continuous user assessment \\
\cline { 2 - 3 } & Customer support & Parsing, sampling \\
\cline { 2 - 3 } & Documentation & Parsing, contract \\
\cline { 2 - 3 } & Objectivity & Parsing \\
\cline { 2 - 3 } & Price & Expert input \\
\cline { 2 - 3 } & Reliability & Contract \\
\cline { 2 - 3 } & Security & Continuous assessment \\
\cline { 2 - 3 } & Timeliness & Parsing \\
\hline Process criteria & Verifiability & Parsing \\
\cline { 2 - 3 } & Accuracy & Expert input \\
\cline { 2 - 3 } & Amount of data & Sampling, cleansing techniques \\
\hline & Availability & Continuous assessment \\
\hline & Consistent representation & Continuous assessment \\
\cline { 2 - 3 } & Latency & Parsing \\
\hline & Response time & Continuous assessment \\
\hline & & \\
\hline
\end{tabular}

\section{THE EXPERT SELECTION FRAMEWORK}

The building of an expert selection framework was divided into three steps: the first step is defining basic knowledge concepts for which the data quality dimensions could rely on; the second step is defining data quality dimensions in the expert selection, using the knowledge concepts; and the third step is defining measurements of expert-oriented FTA methodology quality. In order to facilitate the understanding of the concepts, all concepts defined in this work have a truth value ranging between 0 and 1 . This decision enables the later use of fuzzy variables [20] during the implementation phase.

\subsection{Knowledge measurement}

In order to measure knowledge, we use four concepts: a learning curve model, knowledge depreciation multiplier, knowledge gap, and effective knowledge. 


\subsubsection{Learning Curve}

Although the original Wright learning curve was an exponential function, critics suggested using other curves, which are more suitable. In this work, we chose to model a learning curve that was inspired by a Gompertz function [10] and is defined in equation (2):

$$
K(x)=e^{-d e^{-c x}}
$$

In which:

- $K(x)$ is the person's knowledge in the area;

- $x$ is the amount of experience (time) in the area;

- $\quad d$ is related to the initial knowledge; and

- $c$ is the learning rate.

The parameter $c$ should be adjusted to scale the learning time to the user's scale (which may be in years, months, weeks, etc.). The parameter $\mathrm{d}$ should be adjusted to inform the default initial knowledge; that is, the common knowledge of the area. At the initial knowledge, $x$ is set to zero andthus, $K(0)=e^{-d}$. The parameters $c$ and $d$ must be set as positive values and, consequently, this Gompertz function forms learning curves as shown in Figure 1 and Figure 2.

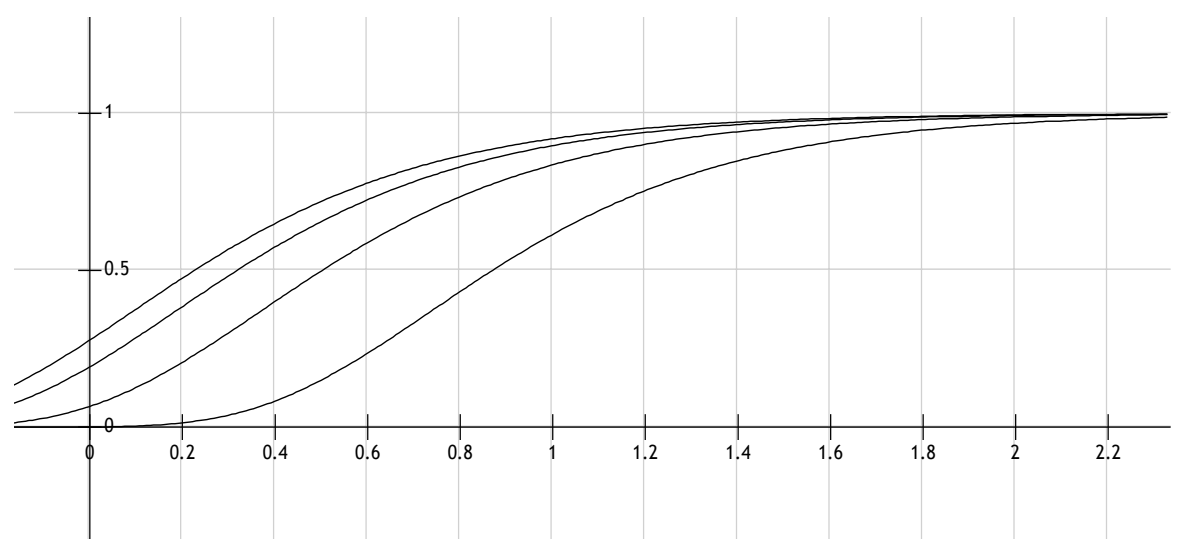

Figure 1.Learning curves in which only parameter $d$ is varied.

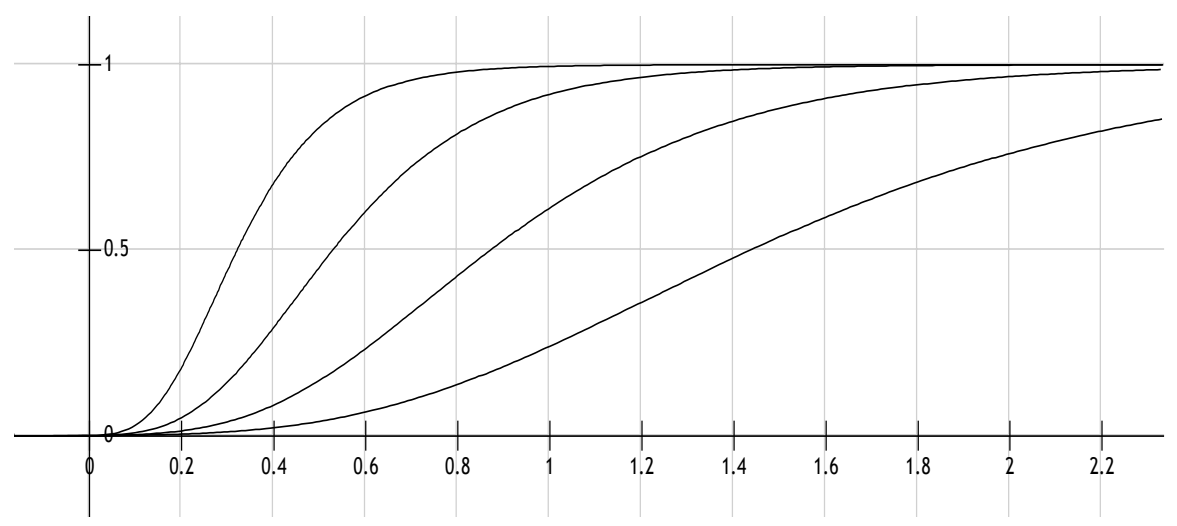

Figure 2.Learning curves in which only parameter $c$ is varied. 


\subsubsection{Knowledge Depreciation (KD) multiplier}

Another fundamental concept which is used in this work is Knowledge Depreciation. We defined a knowledge depreciation (KD) multiplier, which is given as equation (3):

$$
K D(\Delta t)=1-\frac{\ln (r \Delta t+1)}{\ln (r \Delta t+1)+1}
$$

In which:

- $K D$ is the knowledge depreciation multiplier;

- $r$ is the knowledge depreciation (unlearning) rate; and

- $\Delta t$ is the time without learning or practicing the knowledge.

The KD multiplier is the exponential decay in a person's knowledge of a specific area. This factor has a parameter $r$, which indicates the depreciation (unlearning) rate. The higher the value of $r$, the faster the knowledge is depreciated. Moreover, it is important to emphasize that the KD function is asymptotic, which means that a person needs an infinite amount of time to forget everything about some specific knowledge. Another interpretation of the KD multiplier is: What percentage of knowledge is lost once the possible $\mathrm{KD}$ values lie in the interval $[0,1]$ ?

Examples of KD multiplier curves using progressive knowledge depreciation rates $(r)$ are shown in Figure 3.

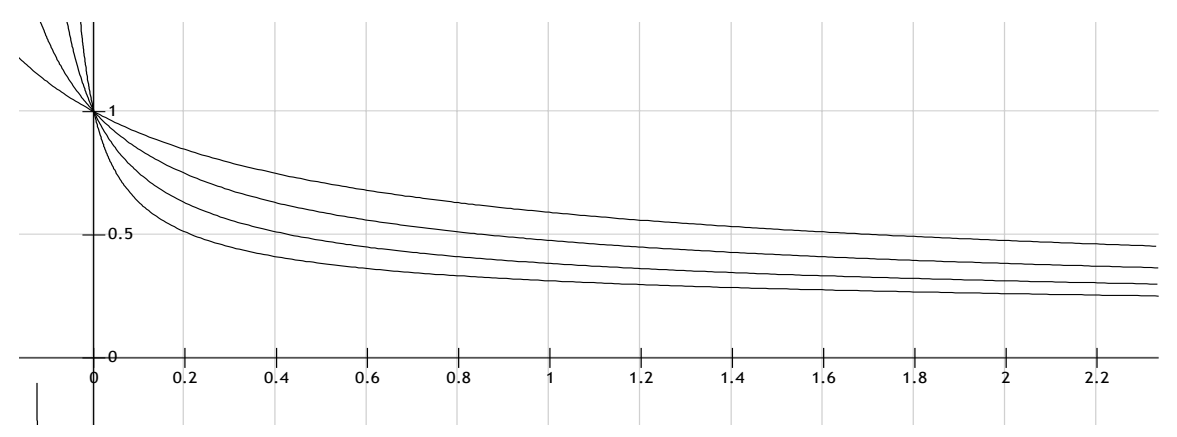

Figure 3.Knowledge Depreciation curves in which the parameter $r$ is varied.

\subsubsection{Knowledge Gap}

The next concept is the Knowledge Gap (KG). The KG measures the amount of knowledge that could be learned if the person didn't stop learning. The KG is defined by equation (4):

$$
K G(x, s)=K(x)-K(s)
$$

In which:

- $K G$ is the person's knowledge gap in the area;

- $x$ is the amount of experience (time) in the area, if learning was not stopped; and

- $s$ is the amount of experience (time) in the area at the moment in which the learning stopped. 
Although the KG concept does not play a fundamental role in this work, it is important to define it and to show its significance in the learning curve graph, as shown in Figure 4.

\subsubsection{Effective Knowledge}

Finally, effective knowledge (EK) is the most important concept in the framework. This concept will be used to transport data quality ideas to the realm of expert selection. EK is defined as the depreciated knowledge, as equation (5) shows:

$$
E K=K(x) K D(x-s)
$$

In which:

- $E K$ is the person's effective knowledge in the area;

- $\quad x$ is the amount of experience (time) in the area, if learning is not stopped; and

- $s$ is the amount of experience (time) in the area at the moment in which the learning stopped.

Due to $\mathrm{KD}$ being defined as exponential decay, as the $\mathrm{x}-\mathrm{s}$ difference gets bigger, the KD multiplier decreases and, thus, the EK becomes lower. Figure 4 shows three curves: the original learning curve; the curve without knowledge depreciation, which stays constant; and the EK, considering the knowledge depreciation as defined in our framework.

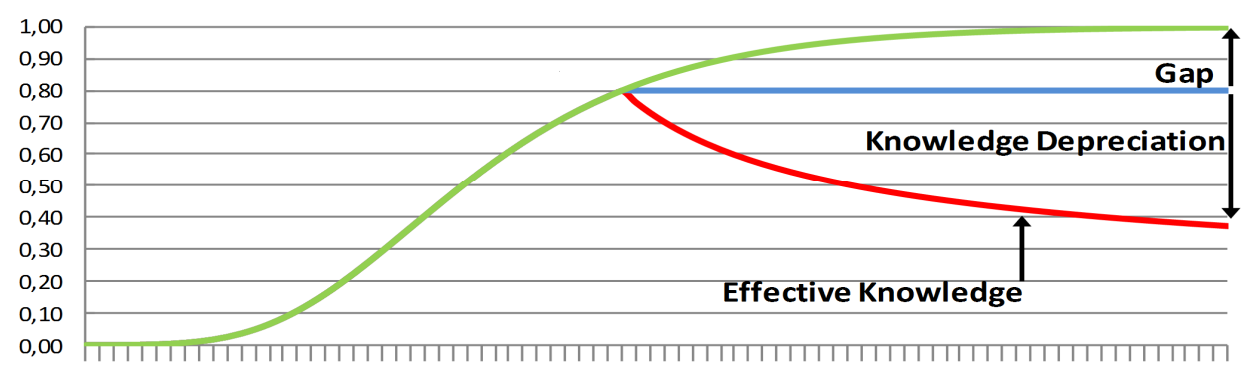

Figure 4. Knowledge Gap, Knowledge Depreciation, and Effective Knowledge.

\subsection{Quality measurement for Expert Selection}

In order to measure an expert selection, we use three data quality dimensions: accuracy, relevancy, and consistency. The data quality concepts were redefined to suit the expert selection framework and they depend only on the EK of the experts. It is important to remember that the EK lies in the $[0,1]$ interval, which means that these dimensions lie in the same interval.

\subsubsection{Accuracy}

The first dimension to be defined in terms of EK is accuracy. Starting with the assumption that accuracy is the "extent to which data are correct", in accordance with Table 3 , accuracy is the mean effective knowledge available, as defined in equation (6):

In which:

$$
\text { Accuracy }=\frac{\sum E K}{n}
$$


- $E K$ is the effective knowledge in the area; and

- $n$ is the number of selected experts.

\subsubsection{Relevancy}

The second dimension is relevancy, which degrades faster than accuracy, especially if EK values are more accurate (i.e., closer to the mean value). At the maximum variation in EK values, relevancy overlaps accuracy, but never surpasses it. Relevancy is defined in equation (7):

$$
\text { Relevancy }=\frac{\sum E K^{2}}{n}
$$

In which:

- $E K$ is the person's effective knowledge in the area; and

- $n$ is the number of selected experts

\subsubsection{Consistency}

The third dimension is consistency, which measures the variation of the EK values. This result was achieved using the EK's standard deviation. We define the consistency dimension in equation (8):

$$
\text { Consistency }=1-2 \sqrt[2]{\frac{\sum E K^{2}-\frac{\left(\sum E K\right)^{2}}{n}}{n}}
$$

In which:

- $E K$ is the person's effective knowledge in the area; and

- $n$ is the number of selected experts.

However, it is possible to replace the terms of equation (8) with the terms of equations (6) and (7), and reinterpret the consistency dimension. This shows that, in this framework, consistency is related to relevancy and accuracy. The result is equation (9):

$$
\text { Consistency }=1-2 \sqrt[2]{\text { Relevancy }- \text { Accuracy }^{2}}
$$

\section{Case Study}

In order to measure the framework's capability to estimate the effective knowledge of an expert panel, the parameters $c$ and $d$ from equation (2), and the parameter $r$ from equation (3), need to be estimated.

Thus, the experiment is divided into two phases: definition of parameters, and examples of the expert selection technique.

\subsection{Definition of parameters}

The first experiment is designed to produce the initial parameters $c, d$, and $r$, which are then used further. We selected 10 experts from our institution and asked 7 students to analyse their curricula available in Lattes Platform [21] - the Brazilian government's platform which integrates researcher's curricula, Research Groups, and Academic Institutions in a single information system. They had to analyse each expert's curriculum, in order to find publications or other proof of activity in the research subject and thus determine: 
International Journal of Database Management Systems ( IJDMS ) Vol.7, No.6, December 2015

- Activity time in one specific area, in years (AcT);

- Inactivity time in the same area, in years (InT);

- Knowledge when leaving the area, in $[0,1]$ interval (MaxK);

- Actual knowledge or effective knowledge (EK) in the area.

Computer supported collaborative work (CSCW) was the chosen area for this part of the experiment. The data collected was grouped in Table 5.

Table 5. Data collected from students.

\begin{tabular}{|c|c|c|c|c|c|c|c|c|}
\hline \multirow{2}{*}{$\begin{array}{c}\text { Expert } \\
\text { number }\end{array}$} & \multicolumn{2}{|c|}{ AcT } & \multicolumn{2}{c|}{ InT } & \multicolumn{2}{c|}{ MaxK } & \multicolumn{2}{c|}{ EK } \\
\cline { 2 - 9 } & Avg & $\sigma$ & Avg & $\sigma$ & Avg & $\sigma$ & Avg & $\sigma$ \\
\hline $\mathbf{1}$ & 18.43 & 1.72 & 2.86 & 0.38 & 0.84 & 0.13 & 0.68 & 0.19 \\
\hline $\mathbf{2}$ & 10.43 & 6.53 & 7.00 & 6.19 & 0.63 & 0.22 & 0.47 & 0.30 \\
\hline $\mathbf{3}$ & 25.14 & 2.41 & 1.14 & 3.02 & 1.00 & 0.00 & 0.97 & 0.08 \\
\hline $\mathbf{4}$ & 9.00 & 2.83 & 1.57 & 2.44 & 0.69 & 0.10 & 0.63 & 0.13 \\
\hline $\mathbf{5}$ & 11.14 & 1.21 & 0.29 & 0.49 & 0.81 & 0.13 & 0.79 & 0.17 \\
\hline $\mathbf{6}$ & 27.86 & 7.60 & 1.29 & 3.40 & 0.97 & 0.08 & 0.94 & 0.11 \\
\hline $\mathbf{7}$ & 6.57 & 2.64 & 5.57 & 3.60 & 0.59 & 0.20 & 0.36 & 0.32 \\
\hline $\mathbf{8}$ & 9.43 & 3.51 & 1.57 & 1.13 & 0.73 & 0.27 & 0.64 & 0.27 \\
\hline $\mathbf{9}$ & 14.75 & 4.45 & 2.66 & 1.70 & 0.78 & 0.15 & 0.68 & 0.19 \\
\hline $\mathbf{1 0}$ & 14.75 & 4.24 & 2.66 & 1.86 & 0.78 & 0.09 & 0.68 & 0.10 \\
\hline
\end{tabular}

In the next step, the data was plotted in a graph and the learning curve (Figure 5) and the knowledge depreciation curve (Figure 6) were interpolated.

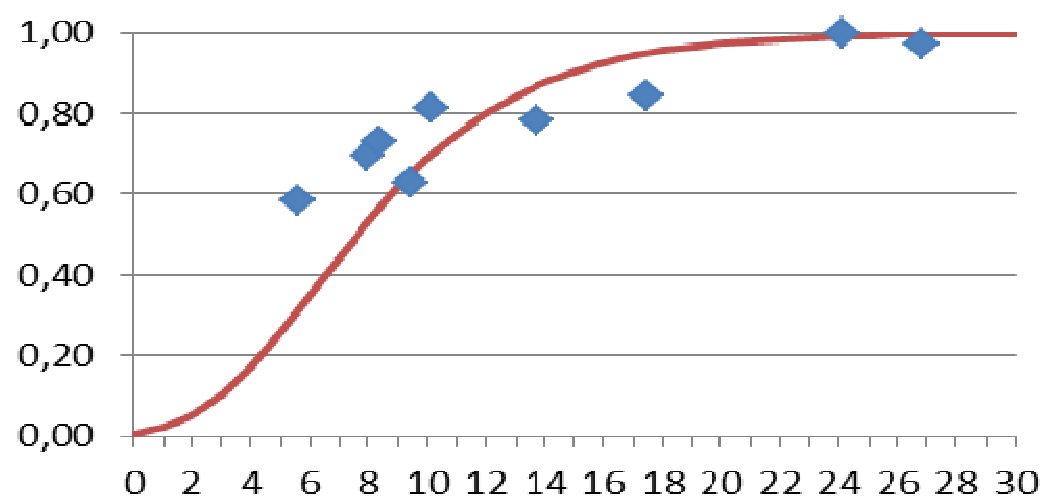

Figure 5.Learning curve from the first experiment.

Analysing the curve patterns, the parameters $d=5, c=0.26$, and $r=0.07$ were chosen as the best fit. It is important to note that these parameters may vary; that is, other curves may have as good a fit as this one. The lack of non-experts in this experiment (people with less than 7 years of experience) made the plotting harder at the beginning of the curve. To solve this problem, we fixed the value for the parameter $d$ to 5, in order to force the initial knowledge to be zero. Then, the $c$ value, which minimizes the total distance of the data points to the curve, was calculated using iterative numerical methods. The parameter $d$ was calculated using the same method as for parameter $c$. 
International Journal of Database Management Systems ( IJDMS ) Vol.7, No.6, December 2015

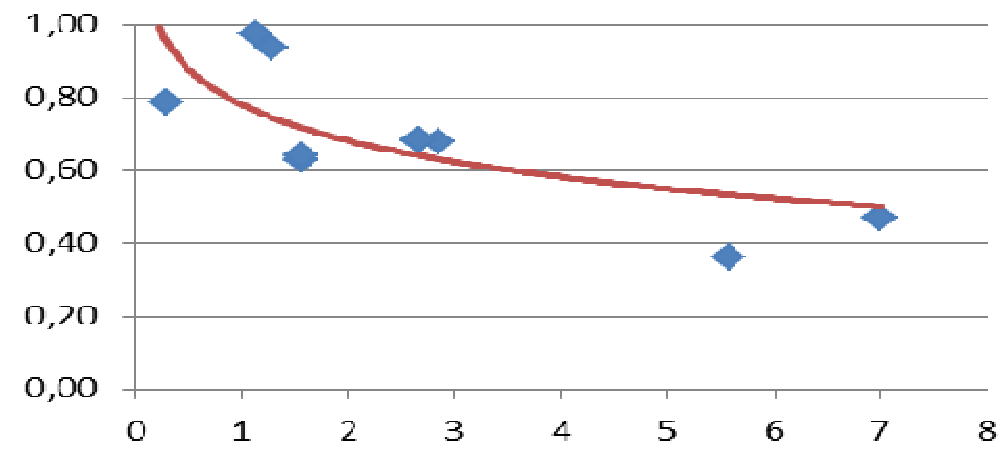

Figure 6. Knowledge depreciation for the first experiment.

Using the resulting data form the experiment, the depreciation curve of $r=0.07$ was plotted to highlight the significance of the depreciation curve. The depreciation curve, limited to a 10 -year window, is shown in Figure 7.

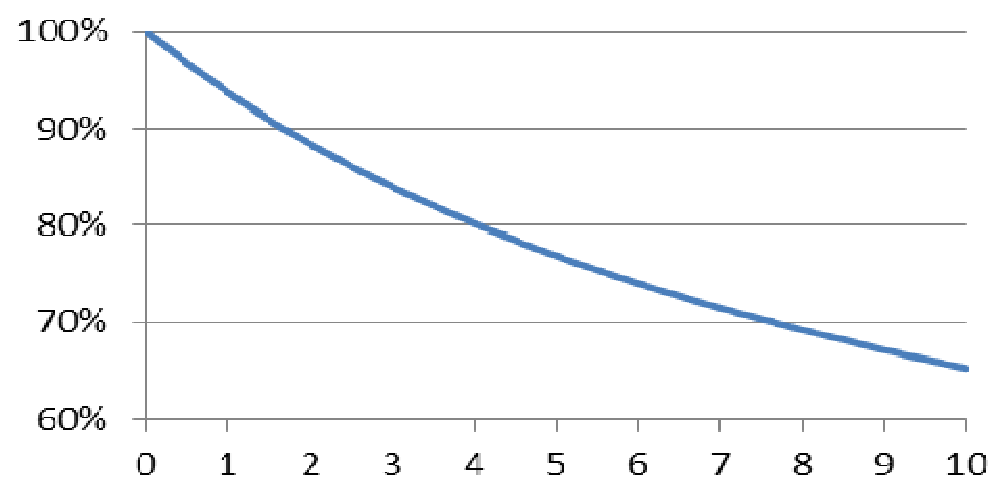

Figure 7. Knowledge depreciation in a 10-year window.

Analysing the depreciation curve, the proposed model indicates that an expert keeps only $65 \%$ of the original knowledge after 10 years of inactivity. This conclusion is made because the value of the function $\mathrm{KD}(10)=0.65$.

\subsection{Examples of expert selection}

The second experiment is a practical application of the expert selection framework. The objective in this experiment is to select two groups of five experts from an initial group of thirty candidates. The size of the initial expert group and the subset to be selected by the algorithm are completely arbitrary. In fact, this algorithm may be used to only rank the experts. The expert areas chosen to be used in this experiment were knowledge management and administration.

In the first step of the algorithm, the candidates were chosen ad hoc. In the second step, their EK in the two areas was measured. Seven students studied their curricula in Lattes Platform [21] to identify their activity time (AcT) and inactivity time (InT) in each area. We computed the average AcT and InT, as well as the standard deviation. With these average AcT and InT values, equations (2), (3) and (5) were used to estimate the EK of each expert in each area, with parameters from the first experiment $(d=5, c=0.26$, and $r=0.07)$. The results are shown in Table 6. 
International Journal of Database Management Systems ( IJDMS ) Vol.7, No.6, December 2015

In the third step, a final list of selected experts was made for each area, by selecting the experts with higher EK. In this theoretical example, there is no risk of experts refusing to participate. The lists for each area, ranked by expertise (the estimated EK), are shown in Table 7 and Table 8 In the next step, the expert selection quality was measured. To achieve the objective, the accuracy, relevancy, and consistency of both expert selections were calculated. The results are shown in Table 9.

According to our framework, the expert selection based on administration was slightly better than the expert selection based on knowledge management, since it has higher accuracy and relevancy. However, its consistency was a little bit lower, which means that the experts have higher EK variation.

Table 6.Activity, inactivity, and estimated effective knowledge.

\begin{tabular}{|c|c|c|c|c|c|c|c|c|c|c|}
\hline \multirow{3}{*}{ Expert } & \multicolumn{5}{|c|}{ Knowledge management } & \multicolumn{5}{|c|}{ Administration } \\
\hline & \multicolumn{2}{|c|}{ AcT } & \multicolumn{2}{|c|}{ InT } & \multirow{2}{*}{ Est. EK } & \multicolumn{2}{|c|}{ AcT } & \multicolumn{2}{|c|}{ InT } & \multirow{2}{*}{ Est. EK } \\
\hline & AVG & $\sigma$ & AVG & $\sigma$ & & AVG & $\sigma$ & AVG & $\sigma$ & \\
\hline 1 & 12.4 & 2.3 & 1.3 & 1.5 & 0.76 & 15.7 & 8.0 & 3.7 & 4.6 & 0.75 \\
\hline 2 & 17.4 & 6.1 & 2.1 & 1.9 & 0.83 & 28.1 & 8.3 & 5.7 & 6.1 & 0.75 \\
\hline 3 & 14.4 & 5.8 & 1.9 & 2.7 & 0.79 & 14.1 & 6.3 & 6.4 & 6.2 & 0.64 \\
\hline 4 & 11.4 & 1.4 & 0.3 & 0.5 & 0.76 & 10.6 & 5.0 & 1.4 & 3.8 & 0.66 \\
\hline 5 & 7.4 & 2.2 & 5.9 & 1.7 & 0.36 & 8.4 & 2.6 & 6.9 & 1.8 & 0.41 \\
\hline 6 & 8.0 & 5.6 & 7.1 & 3.8 & 0.38 & 15.9 & 8.3 & 4.6 & 3.8 & 0.72 \\
\hline 7 & 17.6 & 11.4 & 1.1 & 2.3 & 0.88 & 22.6 & 9.6 & 2.3 & 4.5 & 0.86 \\
\hline 8 & 14.1 & 2.0 & 0.1 & 0.4 & 0.87 & 13.7 & 6.5 & 1.0 & 1.7 & 0.81 \\
\hline 9 & 12.1 & 3.0 & 2.1 & 2.0 & 0.71 & 10.6 & 5.7 & 2.3 & 3.2 & 0.63 \\
\hline $\mathbf{1 0}$ & 12.7 & 1.8 & 2.0 & 1.5 & 0.74 & 7.7 & 7.8 & 8.0 & 9.1 & 0.35 \\
\hline 11 & 6.7 & 1.6 & 1.3 & 0.5 & 0.38 & 10.7 & 5.1 & 13.0 & 15.1 & 0.45 \\
\hline 12 & 4.6 & 9.5 & 6.7 & 4.6 & 0.16 & 14.4 & 8.5 & 4.0 & 4.0 & 0.71 \\
\hline 13 & 11.9 & 1.5 & 1.1 & 1.2 & 0.74 & 22.6 & 16.4 & 1.0 & 1.7 & 0.92 \\
\hline 14 & 3.1 & 1.1 & 9.9 & 0.4 & 0.07 & 7.3 & 6.9 & 2.9 & 4.6 & 0.40 \\
\hline 15 & 13.3 & 3.9 & 1.9 & 2.3 & 0.76 & 16.6 & 2.4 & 1.9 & 2.7 & 0.83 \\
\hline 16 & 20.7 & 7.1 & 1.3 & 2.2 & 0.90 & 17.9 & 12.4 & 1.4 & 1.8 & 0.87 \\
\hline 17 & 2.7 & 1.6 & 8.7 & 2.8 & 0.06 & 11.4 & 6.0 & 10.1 & 11.7 & 0.50 \\
\hline 18 & 22.3 & 8.8 & 4.9 & 5.8 & 0.76 & 18.6 & 7.9 & 5.0 & 7.9 & 0.74 \\
\hline 19 & 13.3 & 4.2 & 0.6 & 0.8 & 0.82 & 21.9 & 5.6 & 0.3 & 0.5 & 0.96 \\
\hline 20 & 9.6 & 8.2 & 3.4 & 3.4 & 0.54 & 19.6 & 7.9 & 1.9 & 3.3 & 0.86 \\
\hline 21 & 10.4 & 5.3 & 1.9 & 2.2 & 0.64 & 21.9 & 5.8 & 1.0 & 1.7 & 0.92 \\
\hline 22 & 14.8 & 2.1 & 0.4 & 0.5 & 0.87 & 14.4 & 2.6 & 1.1 & 1.6 & 0.83 \\
\hline 23 & 15.1 & 4.4 & 0.0 & 0.0 & 0.91 & 20.4 & 4.3 & 0.7 & 1.3 & 0.93 \\
\hline 24 & 14.4 & 4.9 & 1.0 & 2.2 & 0.83 & 35.9 & 5.8 & 1.3 & 2.2 & 0.92 \\
\hline 25 & 5.4 & 6.6 & 2.0 & 1.7 & 0.26 & 18.7 & 5.7 & 3.3 & 4.6 & 0.80 \\
\hline 26 & 8.3 & 2.1 & 0.3 & 0.8 & 0.55 & 8.4 & 3.9 & 2.4 & 3.3 & 0.49 \\
\hline 27 & 7.7 & 3.7 & 0.6 & 1.1 & 0.49 & 10.4 & 3.6 & 2.0 & 2.1 & 0.63 \\
\hline 28 & 8.6 & 6.2 & 5.7 & 3.9 & 0.44 & 22.1 & 3.2 & 0.0 & 0.0 & 0.98 \\
\hline 29 & 16.7 & 8.7 & 1.7 & 2.4 & 0.84 & 26.1 & 4.8 & 3.6 & 3.8 & 0.81 \\
\hline 30 & 14.9 & 2.7 & 0.3 & 0.8 & 0.88 & 19.3 & 12.4 & 1.1 & 2.0 & 0.90 \\
\hline
\end{tabular}


International Journal of Database Management Systems ( IJDMS ) Vol.7, No.6, December 2015

Table 7.Selected experts in knowledge management.

\begin{tabular}{|c|c|c|c|c|}
\hline Rank & Expert & AcT & InT & EK \\
\hline $\mathbf{1}$ & 23 & 15.1 & 0.0 & 0.91 \\
\hline $\mathbf{2}$ & 16 & 20.7 & 1.3 & 0.90 \\
\hline $\mathbf{3}$ & 30 & 14.9 & 0.3 & 0.88 \\
\hline $\mathbf{4}$ & 7 & 17.6 & 1.1 & 0.88 \\
\hline $\mathbf{5}$ & 22 & 14.8 & 0.4 & 0.87 \\
\hline
\end{tabular}

Table 8.Selected experts in administration.

\begin{tabular}{|c|c|c|c|c|}
\hline Rank & Expert & AcT & InT & EK \\
\hline $\mathbf{1}$ & 28 & 22.1 & 0.0 & 0.98 \\
\hline $\mathbf{2}$ & 19 & 21.9 & 0.3 & 0.96 \\
\hline $\mathbf{3}$ & 23 & 20.4 & 0.7 & 0.93 \\
\hline $\mathbf{4}$ & 13 & 22.6 & 1.0 & 0.92 \\
\hline $\mathbf{5}$ & 21 & 21.9 & 1.0 & 0.92 \\
\hline
\end{tabular}

Table 9.Expert selection quality.

\begin{tabular}{|l|c|c|c|}
\hline Expert selection & Accuracy & Relevancy & Consistency \\
\hline Knowledge management & 0.89 & 0.79 & 0.97 \\
\hline Administration & 0.94 & 0.89 & 0.95 \\
\hline
\end{tabular}

\section{Conclusions}

This work presented a data quality framework for forming a panel of experts. The proposed framework defined a method for measuring effective knowledge, based on the learning curve and knowledge depreciation. The effective knowledge may be used to measure the accuracy, relevancy, and consistency of a group of experts; that is, the inherent quality of them.

The experiments achieved their objectives: we successfully estimated the effective knowledge of the experts, and we were able to select experts. The intrinsic quality of the selection was also successfully measured.

\section{ACKNOWLEDGEMENTS}

We would like to thank CAPES and CNPq for their financial support of this work. 
International Journal of Database Management Systems ( IJDMS ) Vol.7, No.6, December 2015

\section{REFERENCES}

[1] A. L. Porter, W. B. Ashton, G. Clar, J. F. Coates, K. Cuhls, S. W. Cunningham, K. Ducatel, P. van der Duin, L. Georgehiou, T. Gordon, H. Linstone, V. Marchau, G. Massari, I. Miles, M. Mogee, A. Salo, F. Scapolo, R. Smits, and W. Thissen, "Technology futures analysis: Toward integration of the field and new methods," Technol. Forecast. Soc. Change, vol. 71, no. 3, pp. 287-303, 2004.

[2] J. Surowiecki, The wisdom of crowds. Anchor, 2005.

[3] T. Uotila and H. Melkas, "Quality of data, information and knowledge in regional foresight processes," Futures, vol. 39, no. 9, pp. 1117-1130, 2007.

[4] R. Johnston, "Historical Review of the Development of Future-Oriented Technology Analysis," in Future-Oriented Technology Analysis, C. Cagnin, M. Keenan, R. Johnston, F. Scapolo, and R. Barré, Eds. Springer Berlin Heidelberg, 2008, pp. 17-23.

[5] V. W. Ruttan, Is war necessary for economic growth? Military procurement and technology development. Oxford University Press, USA, 2006.

[6] A. L. Porter, "Tech forecasting an empirical perspective," Technol. Forecast. Soc. Change, vol. 62, no. 1-2, pp. 19-28, 1999.

[7] T. P. Wright, "Factors affecting the cost of airplanes," J. Aeronaut. Sci. Inst. Aeronaut.Sci., vol. 3, 1936.

[8] L. Argote and D. Epple, "Learning Curves in Manufacturing," Science, vol. 247, no. 4945, pp. 920924, Feb. 1990.

[9] J. R. Dorroh, T. R. Gulledge Jr., and N. K. Womer, "A generalization of the learning curve," Eur. J. Oper. Res., vol. 26, no. 2, pp. 205-216, 1986.

[10] E. Tjørve, "Shapes and functions of species-area curves: a review of possible models," J. Biogeogr., vol. 30, no. 6, pp. 827-835, 2003.

[11] G. Li and S. Rajagopalan, "A learning curve model with knowledge depreciation," Eur. J. Oper. Res., vol. 105, no. 1, pp. 143-154, 1998.

[12] D. E. Rose and D. Levinson, "Understanding user goals in web search," in Proceedings of the 13th international conference on World Wide Web, New York, NY, USA, 2004, pp. 13-19.

[13] A. Dedeke, "A Conceptual Framework for Developing Quality Measures for Information Systems," in Proceedings of the 2000 Conference on Information Quality (IQ-2000), Cambridge, MA, USA, 2000, pp. 126-128.

[14] T. C. Redman, Data quality: the field guide. Access Online via Elsevier, 2001.

[15] Y. Wand and R. Y. Wang, "Anchoring data quality dimensions in ontological foundations," Commun ACM, vol. 39, no. 11, pp. 86-95, 1996.

[16] R. Y. Wang and D. M. Strong, "Beyond accuracy: what data quality means to data consumers," $J$ Manage InfSyst, vol. 12, no. 4, pp. 5-33, 1996.

[17] F. Naumann and C. Rolker, "Assessment Methods for Information Quality Criteria," in Proceedings of the International Conference on Information Quality (IQ), 2000.

[18] M. L. Puri and D. A. Ralescu, "Fuzzy random variables," J. Math.Anal.Appl., vol. 114, no. 2, pp. 409422, 1986.

[19] CNPq - National Council for Scientific and Technological Development, "Lattes Platform." [Online]. Available: http://buscatextual.cnpq.br/buscatextual/busca.do.

\section{AUTHORS}

Carlos Eduardo Barbosa is a D.Sc. student in the Universidade Federal do Rio de Janeiro (UFRJ), where he researches about FTA. His research interests also include Big Data, Decision-support Systems, Knowledge Management, and Crowdsourcing. He also participated and managed projects in the Brazilian Navy

MarcioAntelio is a D.Sc. student in the Universidade Federal do Rio de Janeiro and a associate professor in the Federal Center for Technological Education Celso Suckow da Fonseca. His research interests include Data Quality, Crowdsourcing, and Big Data.

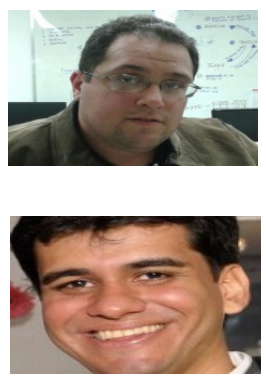


International Journal of Database Management Systems ( IJDMS ) Vol.7, No.6, December 2015

Jano Moreira de Souza is a titular professor of the Universidade Federal do Rio de Janeiro(UFRJ), where he teaches and researches about Knowledge Management, Crowdsourcing, Big Data, Data Quality, Negotiation Support Systems, and Autonomic Computation.

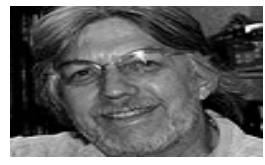

Jonice Oliveira is an associate professor of the Universidade Federal do Rio de Janeiro. Her research interests include Knowledge Management, Social Network Analysis, Competitive Intelligence, Mobile Computing, and Semantic Web.

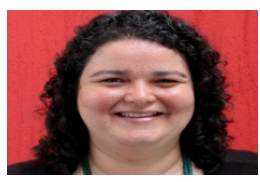

